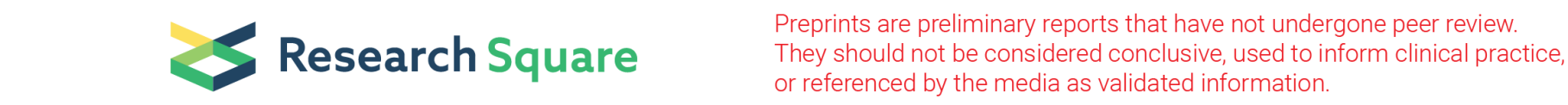

\title{
Diagnostic Value And Safety of Medical Thoracoscopy In The Pleural Effusion of Different Causes
}

\author{
Xiaoting Liu \\ The Second Affiliated Hospital of Xi'an Jiaotong University \\ Xilin Dong ( $\sim \mathrm{dxl1103@sina.com} \mathrm{)}$ \\ The Second Affiliated Hospital of Xi'an Jiaotong University \\ Yu Zhang \\ The Second Affiliated Hospital of Xi'an Jiaotong University \\ Ping Fang \\ The Second Affiliated Hospital of Xi'an Jiaotong University \\ Hongyang Shi \\ The Second Affiliated Hospital of Xi'an Jiaotong University \\ Zongjuan Ming \\ The Second Affiliated Hospital of Xi'an Jiaotong University
}

\section{Research Article}

Keywords: Medicine thoracoscopy, Pleural effusion, Diagnostic value, Safety

Posted Date: July 14th, 2021

DOI: https://doi.org/10.21203/rs.3.rs-668793/v1

License: (9) (i) This work is licensed under a Creative Commons Attribution 4.0 International License. Read Full License

Version of Record: A version of this preprint was published at World Journal of Clinical Cases on April 6th, 2022. See the published version at https://doi.org/10.12998/wjcc.v10.i10.3088. 


\section{Abstract}

Background: Pleural effusions are caused by various reasons, whose diagnosis remains challenging in spite of various means of diagnosis. Medical thoracoscopy, greatly improves the diagnostic efficacy and gets preference for managements, especially undiagnosed pleural effusions. This study aimed to assess the diagnostic efficacy and safety of medical thoracoscopy in patients with pleural effusion of different causes.

Methods: Between January 1st 2012 and April 30th 2021, patients with pleural effusion underwent medical thoracoscopy in the Department of Respiratory Medicine, the Second Affiliated Hospital of Xi'an Jiaotong University. According to the discharge diagnosis, patients were grouped into three, including malignant, tuberculous and inflammatory group. General information, tuberculosis-related and effusion-related indices of three groups were analyzed. The diagnostic yield, diagnostic accuracy, performance under thoracoscopy and complications of patients were compared in three groups. Then, the significant factors for predictive diagnosis between the malignant and tuberculous group were analyzed.

Results: During this 10-year study, 106 patients were included, with 67 males and 39 females, mean age $57.1 \pm 14.184$ years. In 74 patients confirmed under thoracoscopy, 41 patients (38.7\%) were malignant, 21 patients (19.8\%) tuberculous and 32 patients (30.2\%) undiagnostic. The diagnostic yield of medical thoracoscopy is $69.8 \%$, and $75.9 \%$ in the malignant, $48.8 \%$ in the tuberculous, and $75.0 \%$ in the inflammatory. The diagnostic accuracies are $100 \%, 87.5 \%$, and $75.0 \%$, respectively. Under thoracoscopy, we observed single or multiple pleural nodules in $81.1 \%$, pleural adhesions in $34.0 \%$ of patients with pleural effusions. The most common complication was chest pain (41.5\%), following by chest tightness (11.3\%), fever (10.4\%). Multivariate logistic regression analysis showed that effusion appearance $(O R=0.001,95 \% \mathrm{Cl}$ : $0.000-0.204, P=0.010)$, CEA $(O R=0.243,95 \% \mathrm{Cl}$ : $0.081-0.728, P=0.011)$ were significant in the differentiation of malignant and tuberculous pleural effusion.

Conclusion: Medical thoracoscopy is an effective, safe, less invasive procedure with high diagnostic yield for the pleural effusion of different causes. Medical thoracoscopy has a promising prospect.

\section{Background}

Pleural effusion, an abnormal build-up of fluid in the pleural space[1], is a common clinical symptom, caused by cancers, tuberculous pleurisy, inflammation, and organ dysfunction such as heart, liver and kidney[2]. The main manifestation of patients is dyspnea, and other presenting manifestations are largely determined by the underlying diseases. In former times, pleural effusion was diagnosed mainly by clinical history, physical examination, imaging techniques, thoracentesis, percutaneous pleural biopsy. Nevertheless, these methods have low diagnostic yield and delayed diagnosis for pleural effusion, which were associated with markedly higher morbidity and mortality. Currently, medical thoracoscopy (MT), a slightly more invasive procedure and highlighting its efficiency, safety, easy operation, and low price, has a distinctive advantage in diagnosis and treatment improvements in pleural effusion and pleural diseases[3]. Nowadays, it has been well documented to be the gold standard for the diagnosis of pleural effusion[4]. Our study collected relevant clinical data of patients in the Second Affiliated Hospital of Xi'an Jiaotong University, who underwent MT for diagnosis and/or treatment. We evaluated the diagnostic value and safety of MT through analyzing the diagnostic yield and complications in patients with pleural effusion of different causes.

\section{Materials And Methods}

\subsection{Study population}

The study protocol was approved by the Ethics Committee of the Second Affiliated Hospital of Xi'an Jiaotong University. The patients in our institute from January 1st, 2012, to April 30th, 2021, were admitted to the Department of Respiratory Medicine in order to MT for diagnosing and/or treating pleural effusion. Inclusion criteria: 1) pleural effusion was confirmed by chest computerized tomography (CT) scan before admission or before thoracoscopy; 2) undiagnosed pleural effusion (UPE) that cannot be determined by various methods such as thoracentesis, closed pleural biopsy (CPB), bronchoscopy or those who have been diagnosed but need thoracoscopy to treat diseases; and 3) patients undergoing twice MT only collected the first MT data, and all patients underwent pathological tissue biopsy under MT. Exclusion criteria: 1) incomplete clinical data; 2) no pleural space, extensive pleural adhesions, late empyema; 3) poor physical condition, accompanying with severe cardiopulmonary insufficiency, and unable to accept thoracoscopy; and 4) severe hyperemia, bleeding tendency, refractory cough. 


\subsection{Study methods}

The general information of patients includes: age, gender, length of hospitalization, length of time from onset to hospitalization, history of smoking, tumors (personal and family), tuberculosis; history of chronic diseases including hypertension, diabetes mellitus, chronic coronary and lung diseases, others; tumor-related biomarkers such as carcinoembryonic antigen (CEA), neuron-specific enolase (NSE), carbohydrate antigen 125 (CA125), squamous cell carcinoma associated antigen (SCCA), Pro gastrin releasing peptide (PROGPR), Cytokeratin fragment (CYFRA); tuberculosis-related indices such as erythrocyte sedimentation rate (ESR), tuberculosis DNA test, tuberculosis triple antibody test, tuberculosis IgG antibody test, rapid microbial resistance test (X-pert MTB/RIF), tuberculosis infection T lymphocyte spot test (T-SPOT); pleural effusion routine parameters such as counts of nucleated cells, mononucleated cells, and multinucleated cells; pleural effusion biochemical parameters such as lactate dehydrogenase (LDH), protein, glucose, adenosine deaminase (ADA); the position, volume and appearance (bloody, non-bloody), performance under thoracoscopy, pathological results of pleural biopsy, complications. The aetiology of pleural effusion and diagnostic value of MT were analyzed. Patients were grouped into malignant, tuberculous and inflammatory group according to the diagnosis at discharge. The thoracoscopic findings and complications of the three groups were compared. Finally, we analyzed the significant factors for predictive diagnosis between malignant and tuberculous group.

\subsection{Statistical analysis}

All analyses are performed with statistical software (SPSS 18.0). Measurement data not meeting the normal distribution are presented as $\mathrm{M}(\mathrm{Q} 1, \mathrm{Q} 4)$ and Kruskal-Wallis $\mathrm{H}$ test is used for comparison between groups; The enumeration data are presented as $\mathrm{n}(\%)$ and subjected to chi-square test for comparison between groups if bidirectional unordinal variables, noting that more than one expected grid frequency is less than 5, Fisher's exact test is used, or subjected to Kruskal-Wallis $\mathrm{H}$ test if single directional ordinal variables; Logistic regression analysis is used to analyzed the significant factors for predictive diagnosis between malignant and tuberculous group. Variables in Logistic regression analysis were ones that $p$ is less than 0.05 between malignant and tuberculous group. Prior to analysis, the logit converted values need to meet a linear relationship between continuous independent variables and dependent variables, the multiple commonalities need to be excluded between independent and dependent variables. Results were considered statistically significant at $p$ less than 0.05 .

\section{Results}

3.1. General information: Between January 1, 2012, to April 30, 2021, 106 patients with pleural effusions successfully underwent MT, and pleural biopsy samples were obtained for diagnostic evaluation. There were 67 men and 39 women (age range, 21-82 years; mean age, $57.1 \pm 14.184$ years; mean length of hospitalization, $15.57 \pm 5.386$ days; mean time from onset to hospitalization, $57.04 \pm 97.35$ days). In 106 patients, there were 41 (38.7\%) smoking, 12 (11.3\%) personal tumor history, 2 (1.9\%) family tumor history, 2 (1.9\%) tuberculosis history, 41 (38.7\%) chronic disease history, commonly hypertension (11, 10.4\%) and diabetes mellitus (11, 10.4\%). Size of effusion was reported small in 2 (1.9\%), moderate in 32 (30.2\%) and large in 72 (67.9\%). Pleural effusion occurred only on the left side in 31 (29.2\%), only on the right in 52 (49.1\%) and both sides in $23(21.7 \%)$. In 39 (38.8\%) patients, the appearance of pleural effusion was bloody, in $6763.2 \%$ was non-bloody.

In aforementioned indices, age has a statistical difference in the comparison of three groups $(P=0.025)$, which was mainly seen between malignant and tuberculous group; Time in the malignant group was longer than that in the tuberculous and inflammatory group, with $P=0.021$; the incidence of personal tumor history in the malignant group was higher than that in the tuberculous and inflammatory group $(P=0.029)$. In tuberculous indices, the positive rates of tuberculosis DNA, triple antibody, IgG antibody and MTB $\backslash R I F$ were not significantly different in three groups, except for T-SPOT with $P<0.001$ and ESR with $P=0.004$. In tumor biomarkers, CEA, PROGPR, and CYFRA were statistically different in three groups $(P<0.05)$, which were only observed between the malignant and tuberculous group. In effusion-related examinations, LDH, protein, and glucose were not statistically significant in three groups, except for the index of ADA with $P<0.001$. Additionally, counts of nucleated and mononucleated cells in the tuberculous group were more than the others, with $P=0.001$ and $P<0.001$, respectively. Specific data of the patients were shown in Table 1.

Table1囚Characteristics of the study population $(n=106)$ 


\begin{tabular}{|c|c|c|c|c|c|c|c|}
\hline \multicolumn{2}{|l|}{ Variables } & total & Malignant(54) & Tuberculous(43) & Inflammatory(9) & $x^{2} / Z$ & $P$ \\
\hline \multicolumn{2}{|l|}{ Age, yr } & $59(49-68)$ & $62(54-70)^{a}$ & $54(43-66)^{a}$ & $58(51-72)$ & 7.359 & 0.025 \\
\hline \multirow[t]{2}{*}{ Sex } & Male & 67(63.1区 & 34 & 29 & 4 & \multirow[t]{2}{*}{1.723} & \multirow[t]{2}{*}{0.427} \\
\hline & Female & 39(36.8ه & 20 & 14 & 5 & & \\
\hline \multicolumn{2}{|c|}{ Hospital stay(day) } & $\begin{array}{l}15.00(12.00- \\
17.25 \rrbracket\end{array}$ & $\begin{array}{l}16.00(14.00- \\
19.25)^{\mathrm{a}}\end{array}$ & $\begin{array}{l}13.00(11.00- \\
17.00)^{\mathrm{a}}\end{array}$ & $\begin{array}{l}15.00(12.50- \\
23.00)\end{array}$ & 7.773 & 0.021 \\
\hline \multicolumn{2}{|c|}{ Disease duration(day) } & $\begin{array}{l}30.00(15.00- \\
60.00 \rrbracket\end{array}$ & $\begin{array}{l}30.00(20.00- \\
60.00)\end{array}$ & $\begin{array}{l}30.00(10.00- \\
60.00)\end{array}$ & $\begin{array}{l}30.00(8.00- \\
30.00)\end{array}$ & 3.375 & 0.185 \\
\hline \multicolumn{2}{|c|}{ Smoking history } & 41(38.7区 & 19 & 18 & 4 & 0.688 & 0.756 \\
\hline \multicolumn{2}{|c|}{ Personal tumor } & 12(11.3】 & $9^{a}$ & $1^{\mathrm{a}}$ & 2 & 6.815 & 0.029 \\
\hline \multicolumn{2}{|l|}{ Family tumor } & $2(1.9 \rrbracket$ & 2 & 0 & 0 & 1.735 & 0.583 \\
\hline \multicolumn{2}{|c|}{ Tuberculous history } & $2(1.98$ & 0 & 1 & 1 & 4.351 & 0.076 \\
\hline \multicolumn{2}{|l|}{ Hypertension } & 19(17.9ه & 9 & 8 & 2 & 0.431 & 0.930 \\
\hline \multicolumn{2}{|l|}{ Diabetes } & 11(10.4区 & 5 & 4 & 2 & 1.760 & 0.428 \\
\hline \multicolumn{2}{|c|}{ Coronary disease } & $6(5.7 \rrbracket$ & 3 & 3 & 0 & 0.336 & 1.000 \\
\hline \multicolumn{2}{|c|}{ Chronic lung disease } & $3(2.8 \mathrm{D}$ & 0 & 2 & 1 & 4.561 & 0.061 \\
\hline \multicolumn{2}{|c|}{ Other chronic diseases } & $25(23.6 \rrbracket$ & 12 & 10 & 3 & 0.745 & 0.744 \\
\hline \multicolumn{2}{|c|}{ Tuberculosis DNA } & $1(0.9 \rrbracket$ & 0 & 1 & 0 & 2.831 & 0.643 \\
\hline \multicolumn{2}{|c|}{ Tuberculosis triple antibody } & 13(12.3区 & 3 & 10 & 0 & 7.807 & 0.080 \\
\hline \multicolumn{2}{|c|}{ Tuberculosis IgG antibody } & $5(4.7 \rrbracket$ & 2 & 3 & 0 & 4.518 & 0.310 \\
\hline \multicolumn{2}{|l|}{ MTB/RIF } & $4(3.8 \nabla$ & 1 & 1 & 2 & 8.230 & 0.061 \\
\hline \multicolumn{2}{|l|}{ T-SPOT } & $45(42.5 \rrbracket$ & $13^{a}$ & $29^{a}$ & 3 & 19.217 & $\begin{array}{l}\square \\
0.001\end{array}$ \\
\hline \multicolumn{2}{|l|}{$\mathrm{ESR}(\mathrm{mm} \backslash \mathrm{h})$} & $\begin{array}{l}28.00(12.00- \\
44.00 \rrbracket\end{array}$ & $\begin{array}{l}25.50(12.00- \\
39.25)^{a}\end{array}$ & $\begin{array}{l}27.00(12.00- \\
34.00)^{b}\end{array}$ & $\begin{array}{l}62.00(50.00- \\
71.50)^{a, b}\end{array}$ & 11.009 & 0.004 \\
\hline \multirow{3}{*}{$\begin{array}{l}\text { Side of } \\
\text { effusion }\end{array}$} & Left & $31(29.2)$ & 19 & 10 & 2 & \multirow[t]{3}{*}{6.521} & \multirow[t]{3}{*}{0.151} \\
\hline & Right & $52(49.1)$ & 28 & 21 & 3 & & \\
\hline & Bilateral & $23(21.7)$ & 7 & 12 & 4 & & \\
\hline \multirow{3}{*}{$\begin{array}{l}\text { Size of } \\
\text { effusion }\end{array}$} & Small & $2(1.9)$ & 0 & 1 & 1 & \multirow[t]{3}{*}{5.333} & \multirow[t]{3}{*}{0.069} \\
\hline & Moderate & $32(30.2)$ & 12 & 17 & 3 & & \\
\hline & Large & $72(67.9)$ & 42 & 25 & 5 & & \\
\hline \multirow{2}{*}{$\begin{array}{l}\text { Effusion } \\
\text { appearance }\end{array}$} & Bloody & $39(36.8)$ & 29 & 7 & 3 & 14.815 & ( \\
\hline & Non-bloody & $67(63.2)$ & $25^{a}$ & $36^{a}$ & 6 & & \\
\hline CEA(ng\ml) & & $2.22(1.25-4.66)$ & $\begin{array}{l}3.28(1.73- \\
9.10)^{\mathrm{a}}\end{array}$ & $1.40(0.89-2.3)^{\mathrm{a}}$ & $1.45(0.94-4.85)$ & 21.293 & $\begin{array}{l}\square \\
0.001\end{array}$ \\
\hline NSE(ng\ml) & & $\begin{array}{l}16.29(10.96- \\
20.94)\end{array}$ & $\begin{array}{l}\text { 16.44(10.91- } \\
20.80)\end{array}$ & $\begin{array}{l}\text { 16.65(13.00- } \\
23.19)\end{array}$ & $\begin{array}{l}11.40(9.94- \\
14.30)\end{array}$ & 3.934 & 0.140 \\
\hline CA125(U\ml) & & $\begin{array}{l}75.63(33.02- \\
229.63)\end{array}$ & $\begin{array}{l}\text { 65.15(31.64- } \\
231.88)\end{array}$ & $\begin{array}{l}84.89(40.61- \\
285.80)\end{array}$ & $\begin{array}{l}84.73(36.91- \\
112.84)\end{array}$ & 0.830 & 0.660 \\
\hline $\mathrm{SCCA}(\mathrm{ng} \backslash \mathrm{ml})$ & & $0.70(0.50-0.90)$ & $0.70(0.50-$ & $0.70(0.50-1.00)$ & $0.80(0.55-1.15)$ & 1.785 & 0.410 \\
\hline
\end{tabular}


0.80)

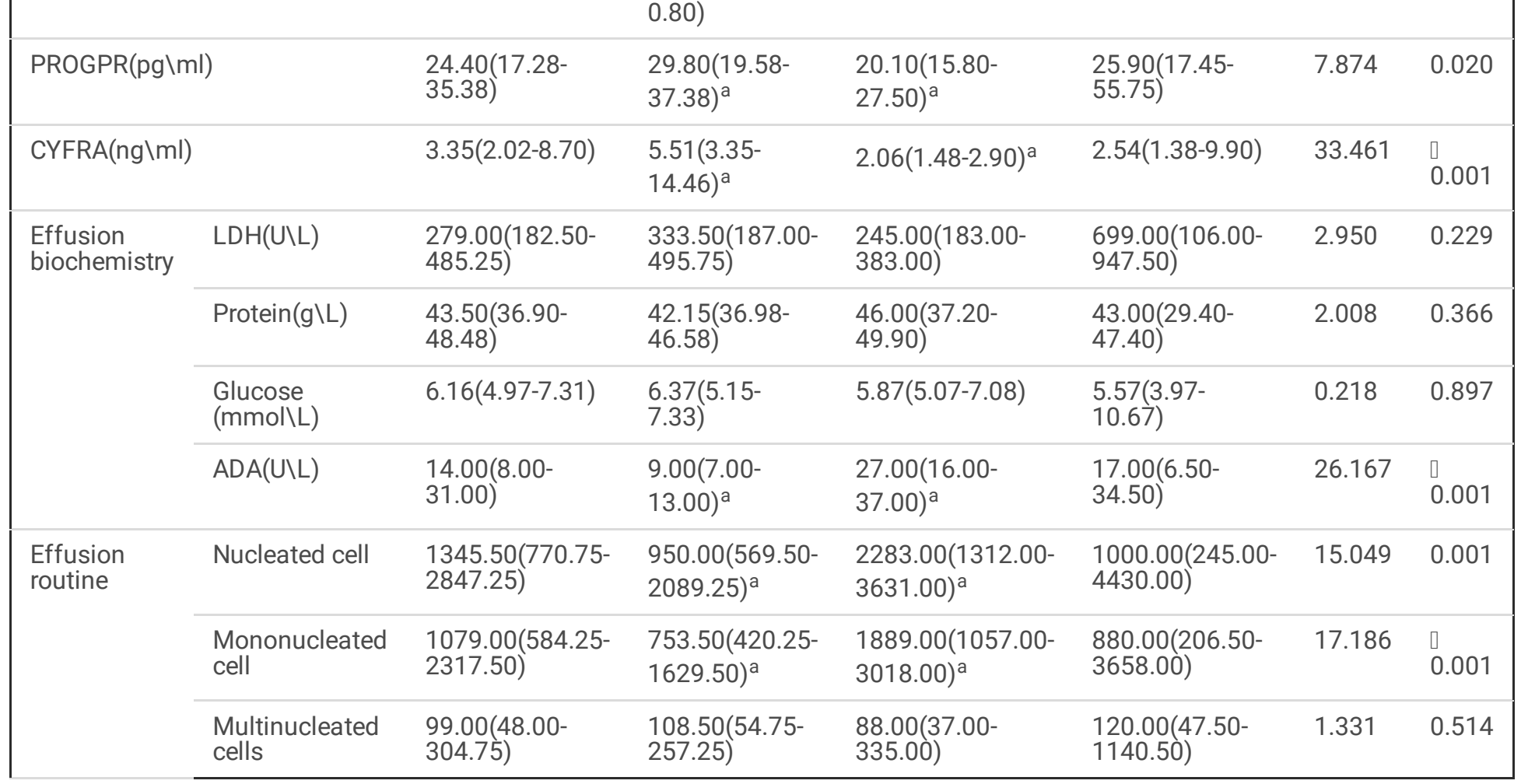

Note: Mean of $\mathrm{a} \square \mathrm{b}$ : the same $\mathrm{a}$ or $\mathrm{b}$ in different groups represents the comparison between the two groups $(P<0.05)$.

3.2. Diagnosis results: In 106 patients, 54 patients were diagnosed as malignant pleural effusion(MPE), 43 patients tuberculous pleural effusion (TBPE), 9 patients inflammatory pleural effusion, according to clinical history, imaging and pleural effusion examination, MT and other inspections. Under MT, 41 patients were confirmed to MPE, consisting of pleural origin in 5, Lung origin in 32 ( 28 adenocarcinomas, 1 squamous carcinoma, 3 small cell carcinomas), non-Lung origin in 4 ( 2 kidney cancer, 1 breast cancer, 1 malignant lymphoma). Therefore, the diagnostic yield of MPE was $75.9 \%$ without misdiagnosed ones, and the diagnostic accuracy was 100\%. In 43 tuberculous patients with pleural effusion, 21 patients were confirmed. The diagnostic yield was $48.8 \%$, yet, 3 exceptions demonstrated inflammation under MT, but the pleural effusion suspected tuberculosis infection and the diagnostic antituberculous chemotherapy was effective, and therefore, the 3 exceptions' diagnoses were subsequently modified as TBPE. Eventually, the diagnostic accuracy was $87.5 \% .12$ patients were confirmed to inflammatory pleural effusion under MT, with 1 purulent pleural effusion, 3 finally diagnosed as TBPE, the diagnostic yield and diagnostic accuracy were $75.0 \%$. Specific data of the patients were seen in Table 2 and 3.

Table 2هEtiological analysis of patients with pleural effusion $(n=106)$ 


\begin{tabular}{|c|c|c|c|}
\hline \multicolumn{2}{|l|}{ Aetiology } & Value & $\%$ \\
\hline \multicolumn{2}{|l|}{ Malignancy } & 41 & $38.7 \rrbracket 41 \backslash 106 \rrbracket$ \\
\hline \multicolumn{2}{|c|}{ Pleural origin } & 5 & $12.2 \bigotimes 5 \backslash 41 \rrbracket$ \\
\hline \multirow[t]{3}{*}{ Lung origin } & Adenocarcinoma & 28 & $68.3 \rrbracket 28 \backslash 41 \rrbracket$ \\
\hline & Squamous carcinoma & 1 & $2.4 \rrbracket 1 \backslash 41 \rrbracket$ \\
\hline & Small cell carcinoma & 3 & $7.4 \llbracket 3 \backslash 41 \rrbracket$ \\
\hline \multicolumn{2}{|c|}{ Non- Lung origin } & 4 & $9.8 \otimes 4 \backslash 41 \rrbracket$ \\
\hline \multicolumn{2}{|l|}{ Tuberculosis } & 21 & $19.8 \otimes 21 \backslash 106 \rrbracket$ \\
\hline \multicolumn{2}{|l|}{ Purulence } & 1 & $0.9 \otimes 1 \backslash 106 \rrbracket$ \\
\hline \multicolumn{2}{|c|}{ Nonspecific inflammation } & 11 & $10.4 \bigotimes 11 \backslash 106 \rrbracket$ \\
\hline \multicolumn{2}{|c|}{ Undiagnosed } & 32 & $30.2 \varangle 32 \backslash 106 \rrbracket$ \\
\hline \multicolumn{2}{|l|}{ Total } & 106 & $100.0 \otimes 106 \backslash 106 \rrbracket$ \\
\hline
\end{tabular}

Table 3囚Diagnostic yield of medical thoracoscopy

\begin{tabular}{|c|c|c|c|c|c|c|c|c|c|c|c|c|}
\hline \multirow[t]{2}{*}{ Aetiology } & \multicolumn{4}{|c|}{ Malignant } & \multicolumn{4}{|c|}{ Tuberculous } & \multicolumn{4}{|c|}{ Inflammatory } \\
\hline & $\mathrm{T}$ & $\mathrm{D}$ & UD & MD & $\mathrm{T}$ & $\mathrm{D}$ & UD & MD & $\mathrm{T}$ & $\mathrm{D}$ & UD & MD \\
\hline $\mathrm{n}$ & 54 & 41 & 13 & 0 & 43 & 21 & 19 & 3 & 9 & 12 & 0 & 3 \\
\hline Diagnostic yield $\mathbb{\%} \otimes$ & \multicolumn{4}{|c|}{75.9} & \multicolumn{4}{|c|}{48.8} & \multicolumn{4}{|c|}{75.0} \\
\hline Accuracy $₫ \% \rrbracket$ & \multicolumn{4}{|c|}{100} & \multicolumn{4}{|c|}{87.5} & \multicolumn{4}{|c|}{75.0} \\
\hline
\end{tabular}

Note $\triangle \mathrm{T}=$ total; $\mathrm{D}=$ diagnosis; $\mathrm{UD}=$ undiagnosis; $\mathrm{MD}=$ Misdiagnosis. Total means total diagnosis numbers at discharge. Diagnosis means diagnosis numbers under MT. undiagnosis means undiagnosis numbers under MT. Misdiagnosis means the diagnosis under MT is inconsistent with the diagnosis at discharge or considered diagnosis after effective management. The inflammation diagnostic yield is calculated as the ratio of the final diagnosed number of 9 at discharge and the number of confirmed diagnosis of 12 under MT, and the diagnostic accuracy is obtained by $100 \%$ subtracting $25.0 \%$ (3\12, misdiagnosis rate).

3.3. Performance under thoracoscopy: Under MT, we observed single or multiple nodules in $86(81.1 \%)$ patients, in which 49 malignant aetiology, 29 tuberculous aetiology, and 8 inflammatory aetiology. In 36 (34.0\%) patients of pleural adhesions, malignant, tuberculous and inflammatory aetiology were $15,15,6$, respectively. Fibrous connective tissue and fibrous bands were seen in $13(12.3 \%)$ patients, consisting of 8 tuberculous and 5 malignant patients. Plaque-like lesions and carbon foam deposition were seen in 11 (10.4\%), consisting of 4 tuberculous and 7 malignant patients. $9(8.5 \%)$ patients demonstrated miliary nodules under MT, mainly observed in the tuberculous group. 6 (5.6\%) patients were focal necrosis, half the malignant and half the tuberculous. 3 (2.8\%) patients with MPE demonstrated neoformation and 2 (1.9\%) patients with TBPE demonstrated pleural thickening. Only one patient confirmed to the malignant demonstrated pleural hyperemia and edema. In the 9 types of thoracoscopic findings above, single or multiple nodules and miliary nodules were statistically significant in the comparison of the three groups $(P<0.05)$. Particularly, single or multiple nodules were more observed in the malignant group than the tuberculous group $(P=0.004)$, whereas miliary nodules in the tuberculous group were more than the malignant group $(P=0.010)$. Specific data were seen in Table 4 .

Table $4 \llbracket$ Thoracoscopic findings $(n=106)$ 


\begin{tabular}{|c|c|c|c|c|c|c|}
\hline Characteristics & $\mathrm{n} \otimes \% \bigotimes$ & Malignant & Tuberculous & Inflammatory & $x^{2}$ & $P$ \\
\hline Single or multiple nodules & $86 \rrbracket 81.1 \rrbracket$ & $49^{a}$ & $29^{a}$ & 8 & 8.335 & 0.011 \\
\hline Miliary nodules & $9 \llbracket 8.5 \rrbracket$ & $1^{\mathrm{a}}$ & $8^{a}$ & 0 & 8.228 & 0.012 \\
\hline Pleural hyperemia and edema & $1 \otimes 0.9 \rrbracket$ & 1 & 0 & 0 & 1.735 & 1.000 \\
\hline Pleural adhesions & $36 \rrbracket 34.0 \rrbracket$ & 15 & 15 & 6 & 4.937 & 0.084 \\
\hline Pleural thickening & $2 \otimes 1.9 \rrbracket$ & 0 & 2 & 0 & 2.656 & 0.326 \\
\hline Fibrous connective tissue and fibrous bands & $13 \otimes 12.3 \rrbracket$ & 5 & 8 & 0 & 2.953 & 0.244 \\
\hline Focal necrosis & $6 \rrbracket 5.6 \rrbracket$ & 3 & 3 & 0 & 0.336 & 1.000 \\
\hline Neoformation & $3 \llbracket 2.8 \rrbracket$ & 3 & 0 & 0 & 2.330 & 0.428 \\
\hline Plaque-like lesions and carbon foam deposition & $11 \otimes 10.4 \rrbracket$ & 7 & 4 & 0 & 0.877 & 0.709 \\
\hline
\end{tabular}

Note: Mean of $\mathrm{a} \square \mathrm{b}$ : the same $\mathrm{a}$ or $\mathrm{b}$ in different groups represents the comparison between the two groups $(P<0.05)$.

3.4 Complications: During this 10-year study, no serious adverse events were recorded in any one patient. local pain was the most common complication in $44(41.4 \%)$ patients, consisting of 21 malignant, 19 tuberculous, 4 inflammatory patients. 12 (11.3\%) patients had chest tightness, consisting of 9 malignant, 1 tuberculous and 2 inflammatory patients. 11 (10.4\%) patients had fever, 7 tuberculous and 4 malignant respectively. 7 (6.6\%) patients had subcutaneous emphysema, 3 tuberculous and 2 malignant, 2 inflammatory, respectively. Bleeding, cutaneous infection at the entry site and prolonged air leak were observed in 2 (1.9\%) patients respectively, 1 malignant and 1 tuberculous. Other complications were mainly nausea in 2, vomiting in 1, arrhythmia (rapid heart rate, rapid atrial fibrillation, frequent atrial fibrillation) in 1 patient. In the 8 complications above, only the incidence of chest tightness was statistically different in three groups $(P<0.05)$, whose difference mainly came from the comparison of the malignant and tuberculous group ( $P=0.039)$. Specific data were seen in Table 5.

Table 5هComplications of thoracoscopy $(n=106)$

\begin{tabular}{|c|c|c|c|c|c|c|}
\hline Complications & $\mathrm{n} \otimes \% \bigotimes$ & Malignant & Tuberculous & Inflammatory & $x^{2}$ & $P$ \\
\hline Fever & $11 \otimes 10.4 \rrbracket$ & 4 & 7 & 0 & 4.403 & 0.250 \\
\hline Bleeding & $2 \otimes 1.9 \rrbracket$ & 1 & 1 & 9 & 0.767 & 1.000 \\
\hline Chest tightness & $12 \varangle 11.3 \rrbracket$ & $9^{a}$ & $1^{\mathrm{a}}$ & 2 & 6.815 & 0.029 \\
\hline Subcutaneous emphysema & $7 \rrbracket 6.6 \rrbracket$ & 2 & 3 & 2 & 3.905 & 0.128 \\
\hline Local pain & $44 \llbracket 41.5 \rrbracket$ & 21 & 19 & 4 & 0.396 & 0.874 \\
\hline Cutaneous infection at the entry site & $2 \otimes 1.9 \bigotimes$ & 1 & 1 & 0 & 0.767 & 1.000 \\
\hline Prolonged air leak & $2 \otimes 1.9 \rrbracket$ & 1 & 1 & 0 & 0.767 & 1.000 \\
\hline Others & $4 \rrbracket 3.8 \rrbracket$ & 2 & 2 & 0 & 0.365 & 1.000 \\
\hline
\end{tabular}

Note: Mean of a $\mathrm{b}$ : the same $\mathrm{a}$ or $\mathrm{b}$ in different groups represents the comparison between the two groups $(P<0.05)$.

3.5. Multivariate analysis of malignant and tuberculous pleural effusion: Owing to the small samples (9 cases) of patients with inflammatory pleural effusion, and statistically difference in three groups mainly focusing on the malignant and tuberculous group, the multivariate analysis was performed only in the malignant and tuberculous group. Variables that $P<0.05$ in univariate analysis were included in the logistic regression model. Prior to analysis, the logit converted values met a linear relationship ( $P>0.05)$ between continuous independent variables and dependent variables. Additionally, multiple commonalities (tolerance $>0.1$ and variance expansion factor $<10$ ) were excluded between independent and dependent variables, therefore, 15 variables met the inclusion criteria. Logistic regression analysis showed that the effusion appearance $(O R=0.001,95 \% \mathrm{Cl}$ : 0.000-0.204, $P=0.010), \mathrm{CEA}(O R=0.243,95 \% \mathrm{Cl}$. 
0.081-0.728, $P=0.011$ ) were statistically significant, that is to say, bloody pleural effusion and CEA played a predictive rule in the differential diagnosis of malignant and tuberculous pleural effusions. Specific data were seen in Table 6.

Table 6囚 Multivariate analysis of malignant and tuberculous group

\begin{tabular}{|llllllll|}
\hline Variables & $\beta$ & $S E$ & $P$ & $O R$ & \multicolumn{2}{l|}{$95 \% \mathrm{Cl}$} \\
\cline { 6 - 8 } & & & & & lower & upper \\
\hline Age, yr & -0.042 & 0.037 & 0.259 & 0.959 & 0.892 & 1.031 \\
\hline Hospital stay(day) & -0.032 & 0.125 & 0.800 & 0.969 & 0.758 & 1.238 \\
\hline Personal tumor & -1.223 & 1.865 & 0.512 & 0.294 & 0.008 & 11.385 \\
\hline T-SPOT & -2.259 & 1.467 & 0.124 & 0.105 & 0.006 & 1.854 \\
\hline ESR & 0.007 & 0.032 & 0.814 & 1.008 & 0.946 & 1.072 \\
\hline Effusion ADA & 0.212 & 0.109 & 0.052 & 1.236 & 0.998 & 1.530 \\
\hline Effusion appearance & -6.710 & 2.613 & 0.010 & 0.001 & 0.000 & 0.204 \\
\hline CEA & -1.413 & 0.559 & 0.011 & 0.243 & 0.081 & 0.728 \\
\hline PROGPR & -0.097 & 0.051 & 0.060 & 0.908 & 0.821 & 1.004 \\
\hline CYFRA & -0.064 & 0.038 & 0.091 & 0.938 & 0.870 & 1.010 \\
\hline Nucleated cell & 0.001 & 0.000 & 0.263 & 1.001 & 1.000 & 1.002 \\
\hline Mononucleated cell & -0.001 & 0.001 & 0.354 & 0.999 & 0.998 & 1.001 \\
\hline Single or multiple nodules & 2.143 & 2.008 & 0.286 & 8.526 & 0.167 & 436.395 \\
\hline Miliary nodules & -1.982 & 2.936 & 0.500 & 0.138 & 0.000 & 43.469 \\
\hline Chest tightness & 5.356 & 2.817 & 0.057 & 211.770 & 0.848 & 52896.349 \\
\hline
\end{tabular}

\section{Discussion}

Pleural effusions are caused by various reasons, whereas the common causes are congestive heart failure, malignancy, pneumonia, and pulmonary embolism[2]. In addition to mesothelioma, pleural metastatic carcinomas from lung, breast, lymphoma are common causes in MPE[5], while in benign pleural effusion, tuberculosis is the most one. As reported, $16.7 \%$ of patients with malignancy probably develops an effusion during their disease[6], with $15 \%$ at presentation and $50 \%$ during their disease in lung cancer[5], $90 \%$ in malignant pleural mesothelioma (MPM)[7]. As regard TBPE, in tuberculosis endemic areas, the incidence of pleural involvement approaches $30 \%$, and $3 \%-5 \%$ in non-endemic areas[8]. In our study for etiological analysis of pleural effusion, malignant origin ranked first (38.7\%), mainly subclassified into pleural metastatic carcinomas originating from lung, then tuberculous origin (19.8\%), finally inflammatory origin (11.3\%). In a meta-analysis involving 2380 patients in the etiological analysis of pleural effusion who underwent MT, revealed that the malignant, tuberculous, and inflammatory causes accounted for $56.2 \%, 21.6 \%$, and $17.5 \%$, respectively. In patients with malignant causes, $37.8 \%$ of patients were metastatic carcinomas, mainly from the lung (22.6\%)[9]. Although the samples in our study are small, the results are similar to those of large samples. Of note, in our study, MPE takes up a considerable proportion, as the majority of patients with MPE had obtained a definitive diagnosis and got the procedure of MT in order to seek a present or subsequent treatment as well as symptomatic relief. Rather, TBPE is less than $20 \%$, since these patients mainly came from UPE in our study, which still leaves a low diagnostic level even under MT as reported[8]. Inflammatory pleural effusion has an unneglectful proportion under MT, due partly to accepting some patients who had no evidences for malignance and tuberculosis except for inflammation.

Differentiating benign from malignant pleural effusions is critical for diagnosis establishment, management guidance, and prognosis judgement[10]. Over the past years, it was the primary methods that effusion examination, CPB, coupled with clinical history, blood biochemistry, imaging examination, are applied to distinguish benign and malignant pleural effusion, despite the low diagnostic yield. Studies have shown that thoracocentesis yielded a diagnosis of pleural effusion in $60 \%$, and CPB in $45 \%$. By contrast, the combined 
diagnostic yield can be improved to $75 \%$. Recently, using thoracic ultrasound and/or CT to provide real-time image guidance has been increasingly and widespreadly adopted which should be the best practice to optimise diagnostic yield and patients' safety, avoid subsequently invasive procedures such as MT, unequivocally backed up by national guidelines[11]. A randomised controlled trial revealed that CT-guided biopsy improved the diagnostic yield of $40 \%$ by comparison with unassisted CPB in patients with MPE ( $87 \%$ vs. 47\%)[12]. In terms of safety, another observational cohort study demonstrated that performing ultrasound-guided thoracentesis could reduce the risk of pneumothorax by $19 \%$ and bleeding complications by $68 \%[13]$. Nonetheless, there are still UPE patients of $8 \%-25 \%[14]$, probably as image-guided biopsy allows limited access to adequate quantities of tissue by comparison with thoracoscopy, particularly for those that additional molecular analysis is required or histological diagnosis is challenging. Fortunately, MT appears to bring revolutionary improvements in the diagnosis and management of pleural effusion, and the diagnostic yield for UPE is reported up to $80 \%-99 \%[15,16]$. Among these 106 patients in our study, MT yielded a diagnosis in 74 of 106 patients (69.8\%), with an undiagnostic yield in $30.2 \%$. A systematic review including 4 articles with each 21-68 patients yielded a diagnosis by MT in patients with UPE, ranging from $66.7 \%$ to $97 \%$. Simultaneously, the study performed an analogic single-centred research in 48 congener patients revealing a diagnostic yield of $66.7 \%$, a proportion similar to ours[17]. However, some reports revealed a higher diagnostic yield of MT that up to $95 \%[18]$. We take into account the development level of MT and operators' technical proficiency in different regions and hospitals. Anyway, regardless of high or low diagnostic yield, the diagnostic sensitivity and specificity of MT are still at a high level[19]. It is noticeable that the diagnostic yield of MT for MPE is relatively high, on the contrary for TBPE. As regards our study for pleural effusion with different aetiologies, the diagnosis yield for malignancy, tuberculosis and inflammation were $75.9 \%$, $48.8 \%, 75.0 \%$, with diagnostic accuracy $100 \%, 87.5 \%$, and $75.0 \%$, respectively. Relevant studies reported the diagnostic yield of malignant and benign pleural effusion, revealing in $65.8 \%, 34.2 \%$ respectively, with a diagnostic accuracy of $97.4 \%[20]$. Another study showed similar results (diagnostic yield for malignant $68.3 \%$, benign $31.7 \%$, and diagnostic accuracy $97.6 \%$ )[21]. To our knowledge, the diagnosis of TBPE is difficult, largely due to the paucibacillary nature of these effusions and low yield on mycobacterium tuberculosis culture, which is on account of the compartmentalization of pleural effusion and effective containment of this bacilli by cytokine milieu[8]. As yet, thoracoscopic pleural pathology has been known as the gold standard method for TBPE diagnosis[22], but tissue material selection is not always available, which therefore limits its diagnosis. Furthermore, the effects of variables on predicting benign and malignant pleural effusion are differential. In our study, it's meaningful of some indices such as age, blood and effusionrelated indices, and effusion appearance in univariate analysis between the benign and malignant group. Whereas, only bloody effusion and CEA have a predictive value after multivariate analysis, which is consistent with clinical practice. However, it is always challenging to distinguish TBPE from MPE due to the lack of specificity of the clinical features, despite some indices such as CEA, LDH, ADA, T-SPOT, mononuclear cells count, gamma interferon (IFN-y), interleukin 12 (IL-12), X-pert MTB/RIF, etc. reported as potential predictors $[8,23,24]$.

Unlike thoracocentesis and $\mathrm{CPB}, \mathrm{MT}$ permits biopsy for suspicious lesions with direct visualization to improve the diagnostic yield of pleural effusion, which can be targeted accurately[25]. In our study, single or multiple nodules (89.6\%) were the most findings under MT, following by pleural adhesions (34.0\%). For MPE, single or multiple nodules (46.2\%), pleural adhesions (14.2\%), plaque-like lesions and carbon foam deposition (6.6\%) were reported successively. For TBPE, in addition to single or multiple nodules (27.4\%) and pleural adhesions (14.2\%), frequent reports were miliary nodules (7.5\%), fibrous connective tissue and fibrous bands (7.5\%). As regards inflammatory pleural effusion, only nodules and pleural adhesions were noticed. Lama et al. reported the thoracoscopic findings of 107 patients with MPE, revealing pleural nodules (81.3\%) and pleural adhesions (40.2\%)[26]. Wang et al. with 333 patients diagnosed as tuberculous pleurisy by MT, revealed pleural nodules (69.4\%), pleural adhesions (66.7\%), hyperemia (60.7\%), and plaque-like lesions (6.0\%)[27]. The common findings in our study are similar to those in previous reports. It can be found that pleural nodules and pleural adhesions are the most frequent pleural abnormalities under MT in both reported researches and our study. Pleural nodules, one of the MT indications, classified as benign and malignant properties, generally were caused by tumors, tuberculosis, and inflammation. Unlike the lung nodules, radiologic methods such as CT often fails to pick up early pleural abnormalities, given a similar density between the apposed pleura and adjacent pleural effusion[10]. Therefore, MT has been the optimal choice, owing to its direct visible access to the lesions. Pleural adhesions refer to the two layers of pleura sticking together, along with pleural thickening if the fibrin in the effusion is deposited on the pleura. The presence of pleural adhesions may prevent full examination of pleural effusions and/or pleural diseases. A retrospective analysis of 540 patients with MPE who underwent MT, found a high frequency of significant adhesions (40\%) and an inverse correlation between the extent of pleural adhesions and the sensitivity of MPE cytology, when the grade of adhesions from 0 to 4, the cytologic sensitivity of MPE from 71\% down to 20\%[28]. Moreover, pleural adhesions can mount the number of thoracentesis. Therefore, pleural adhesions have been challenging the diagnosis and management of pleural effusions and/or pleural diseases. As we know, serious pleural adhesion that leaves no pleural space is an absolute contraindication for MT[29].

Page $9 / 13$ 
In our study, the extent of pleural adhesions permitted conducting MT, that's why our proportion of pleural adhesions is lower than reported literatures. Other findings under MT were less than $15 \%$ in our study. Briefly speaking, representative characteristics of different pleural diseases under MT generally are not distinct; however, visual judgement for differential performance of lesions coupled with professional cognition for diseases can partly help pulmonologists obtain preliminary inference.

MT is now increasingly commonplace in pleural interventional practice, where recent years have seen rapid and unprecedented variations in more rapid access to diagnosis and treatment with yields and safety levels akin to or even surpassing those provided by other methods[11]. To a great extent, its safety should be attributed to the procedure's standard operating specification, such as special semi-rigid instruments that allows a single small skin incision for insertion of a disposable flexible trocar, adequate patient preparation prior to the procedure, local anesthesia, moderate sedation and analgesis, spontaneous ventilation, accompanied by electrocardiographic and oxygen saturation monitoring throughout the procedure[29]. However, it is generally acknowledged that complications are inevitable, and the reasons should be considered in two situations, when the operators have not excellent professional knowledge for anatomical structure of thoracic cavity and proficient operational techniques which require a learning process to master, and when the patients have special physical constitutions. Therefore, careful assessment of patients' condition, adequate training of pulmonologists' operating skills, careful consideration of contraindications and prevention of complications cannot be overemphasized prior to the procedure[18]. The Medicine Thoracoscopy Diagnostic Specifications by Chinese Medical Doctor Association lists 23 possible complications including 3 prior to MT, 7 during MT, and 13 after MT[29]. Unlike that, our study obtained more complications after MT owning to limited data collection methods that relied on electronic medical records. In our retrospective study, the results revealed pain at the entry site (41.5\%), chest tightness slightly (11.3\%), fever (10.4\%), and subcutaneous emphysema (6.6\%), other reported complications less than $5 \%$ which recovered soon after symptomatic treatments, and no one thoracoscopy-related death. A large sample study with 1926 patients with pleural effusion undergoing MT reported the most common complications were pain (38.9\%), fever (20.8\%), cutaneous infection at the entry site (7.1\%), and subcutaneous emphysema (3.2\%), however, the rare complications were prolonged air leakage, bleeding, Lung laceration, pulmonary re-expansion oedema, mediastinal emphysema and mortality, whose incidences were less than $0.5 \%[30]$. By contrast, other recorded complications such as prolonged air leak, cutaneous infection at the entry site, and bleeding in our study were also less than $2 \%$. Collectively, it is generally agreed that MT appears to be relatively safe and deserves clinical promotion vigorously.

This study has several limitations. Firstly, it's a single-centred retrospectively study, whose results are responsible for the population in the local regions and hospitals but will be referential for the population from different regions and hospitals. Secondly, the timespan for sample selection is large and the sample size is small. Our study involved eligible patients from 2012 to 2021, when the technology of MT was on the way of development and maturation in our hospital. At the beginning of development, the limitations such as operators' technical proficiency, equipment configuration, and team's co-ordination, discounted the results of diagnosis and management. It may be why the diagnostic yield of pleural effusion of different causes in our study is lower than that confirmed by MT in the latest literatures. Small samples of patients with inflammatory pleural effusion rendered it unable to conduct multivariate analysis, which will be improved in respect of samples in the later study.

\section{Conclusion}

In conclusion, MT appears to be efficient and relatively safe in the management of pleural diseases. Compared with effusion examination and pleural biopsy, its advantages lie in the factors including higher diagnostic yield and safety, easier use, lower cost and better tolerability to patients, which confer MT a significant clinical value. Presently, the domestic MT has been proficient but is still in limited use and slow uptake by respiratory physicians in non-first-tier cities and non-large hospitals, which has delayed the diagnosis and management of thoracoscopy-adapted diseases. With the rapidly evolving development, it is vital that knowledge of MT is disseminated as widely and as efficiently as possible, and this novel pleural techniques will also usher in more potential benefits.

\section{Abbreviations}

ADA Adenosine deaminase

CA125 Carbohydrate antigen 125

CEA Carcinoembryonic antigen 
CPB Closed pleural biopsy

CT Computerized tomography

CYFRA Cytokeratin fragment

ESR Erythrocyte sedimentation rate

IFN-y Gamma interferon

IL-12 Interleukin 12

LDH Lactate dehydrogenase

MPE Malignant pleural effusion

MPM Malignant pleural mesothelioma

MT Medical thoracoscopy

NSE Neuron-specific enolase

PROGPR Pro gastrin releasing peptide

SCCA Squamous cell carcinoma associated antigen

TBPE Tuberculous pleural effusion

T-SPOT T lymphocyte spot test

UPE Undiagnosed pleural effusion

X-pert MTB/RIF Rapid microbial resistance test method

\section{Declarations}

Acknowledgments

The authors are grateful to the staff at the Department of Respiratory Medicine at the Second Affiliated Hospital of Xi'an Jiaotong University for their non-financial assistance of this study.

Author's contributions

XLD conceived and designed the study. XTL and YZ analyzed the data and collected the related clinical information. XTL participated in the writing of the manuscript content, PF, HYS and ZJM revised the manuscript critically for relevant intellectual content. All authors have endorsed the final version of the manuscript. All authors read and approved the final manuscript.

Funding

This work was supported by Shaanxi Science and Technology Research Plan Program (2020SF-106), Shaanxi, China. The funders had no role in study design, data collection and analysis, or preparation of the manuscript.

Availability of data and materials

The datasets analysed during the current study are available from the corresponding author on reasonable request.

Ethics approval and consent to participate

This study was reviewed and approved by the Ethics Committee of the Second Affiliated Hospital of Xi'an Jiaotong University. Informed consent was waived because this was a retrospectively study. We obtained patient data from the Medical Records. We 
analysed the data anonymously. The use of the raw data was permitted by the Ethics Committee of the Second Affiliated Hospital of Xi'an Jiaotong University, and the approval number was 2021055.

Consent for publication

Not applicable.

Competing interests

The authors declare that they have no competing interests.

Footnotes

Publisher's Note

Springer Nature remains neutral with regard to jurisdictional claims in published maps and institutional affiliations

\section{References}

1. Semaan R, Feller-Kopman D, Slatore C, Sockrider M: Malignant Pleural Effusions. Am J Respir Crit Care Med 2016, 194(6):P11-P12.

2. Jany B, Welte T: Pleural Effusion in Adults-Etiology, Diagnosis, and Treatment. Dtsch Arzteb/ Int 2019, 116(21):377-386.

3. Lee P, Folch E: Thoracoscopy: Advances and Increasing Role for Interventional Pulmonologists. Semin Respir Crit Care Med 2018, 39(6):693-703.

4. Anevlavis S, Froudarakis ME: Advances in pleuroscopy. Clin Respir J 2018, 12(3):839-847.

5. Feller-Kopman D, Light R: Pleural Disease. N Engl J Med 2018, 378(8):740-751.

6. Walker S, Mercer R, Maskell N, Rahman NM: Malignant pleural effusion management: keeping the flood gates shut. Lancet Respir Med 2020, 8(6):609-618.

7. Koegelenberg CFN, Shaw JA, Irusen EM, Lee YCG: Contemporary best practice in the management of malignant pleural effusion. Ther Adv Respir Dis 2018, 12:1753466618785098.

8. Shaw JA, Diacon AH, Koegelenberg CFN: Tuberculous pleural effusion. Respirology 2019, 24(10):962-971.

9. Zhang S, Tian D-H, Liang B, Jiang S-J: Diagnostic value of medical thoracoscopy in $\mathbf{2 3 8 0}$ patients with pleural effusion. The Journal of Practical Medicine. 2013, 29(8):1316-1318.

10. Fysh ETH, Thomas R, Tobin C, Kuok YJ, Lee YCG: Air in the Pleural Cavity Enhances Detection of Pleural Abnormalities by CT Scan. Chest 2018, 153(6):e123-e128.

11. Bhatnagar R, Corcoran JP, Maldonado F, Feller-Kopman D, Janssen J, Astoul P, Rahman NM: Advanced medical interventions in pleural disease. Eur Respir Rev 2016, 25(140):199-213.

12. Maskell NA, Gleeson FV, Davies RJO: Standard pleural biopsy versus CT-guided cutting-needle biopsy for diagnosis of malignant disease in pleural effusions: a randomised controlled trial. Lancet 2003, 361(9366):1326-1330.

13. Mercaldi CJ, Lanes SF: Ultrasound guidance decreases complications and improves the cost of care among patients undergoing thoracentesis and paracentesis. Chest 2013, 143(2):532-538.

14. Agarwal A, Prasad R, Garg R, Verma SK, Singh A, Husain N: Medical thoracoscopy: a useful diagnostic tool for undiagnosed pleural effusion. Indian J Chest Dis Allied Sci 2014, 56(4):217-220.

15. Botana Rial M, Lojo Rodríguez I, Mouronte Roibás C, Leiro Fernández V, Núñez Delgado M, Salgado Barreira Á, Pereira Torrado A, Fernández Villar A: Diagnostic Yield and Safety of Pleural Cryobiopsy during Medical Thoracoscopy to Diagnose Pleural Effusion. A

Page $12 / 13$ 
Systematic Review and Meta-Analysis. Arch Bronconeumo/ 2020, 56(12):784-791.

16. Dixon G, de Fonseka D, Maskell N: Pleural controversies: image guided biopsy vs. thoracoscopy for undiagnosed pleural effusions? J Thorac Dis 2015, 7(6):1041-1051.

17. Nattusamy L, Madan K, Mohan A, Hadda V, Jain D, Madan NK, Arava S, Khilnani GC, Guleria R: Utility of semi-rigid thoracoscopy in undiagnosed exudative pleural effusion. Lung India 2015, 32(2):119-126.

18. Burrows NJ, Ali NJ, Cox GM: The use and development of medical thoracoscopy in the United Kingdom over the past 5 years. Respir Med 2006, 100(7):1234-1238.

19. Mohan A, Chandra S, Agarwal D, Naik S, Munavvar M: Utility of semirigid thoracoscopy in the diagnosis of pleural effusions: a systematic review. J Bronchology Interv Pulmonol 2010, 17(3):195-201.

20. Rozman A, Camlek L, Kern I, Malovrh MM: Semirigid thoracoscopy: an effective method for diagnosing pleural malignancies. Radiol Oncol 2014, 48(1):67-71.

21. Rozman A, Camlek L, Marc-Malovrh M, Triller N, Kern I: Rigid versus semi-rigid thoracoscopy for the diagnosis of pleural disease: a randomized pilot study. Respirology 2013, 18(4):704-710.

22. Lei X, Wang J, Yang Z, Zhou S, Xu Z: Diagnostic Value of Pleural Effusion Mononuclear Cells Count and Adenosine Deaminase for Tuberculous Pleurisy Patients in China: A Case-Control Study. Front Med (Lausanne) 2019, 6:301.

23. Yang $X$, Feng M, Shen Y, Deng B, He Y, Cao G: Clinical characteristics and potential indicators for definite diagnosis of tuberculous pleural effusion. Artif Cells Nanomed Biotechnol 2019, 47(1):1924-1931.

24. Porcel JM: Biomarkers in the diagnosis of pleural diseases: a 2018 update. Ther Adv Respir Dis 2018, 12:1753466618808660.

25. Shaikh F, Lentz RJ, Feller-Kopman D, Maldonado F: Medical thoracoscopy in the diagnosis of pleural disease: a guide for the clinician. Expert Rev Respir Med 2020, 14(10).

26. Sakr L, Maldonado F, Greillier L, Dutau H, Loundou A, Astoul P: Thoracoscopic assessment of pleural tumor burden in patients with malignant pleural effusion: prognostic and therapeutic implications. J Thorac Oncol 2011, 6(3):592-597.

27. Wang Z, Xu L-L, Wu Y-B, Wang X-J, Yang Y, Zhang J, Tong Z-H, Shi H-Z: Diagnostic value and safety of medical thoracoscopy in tuberculous pleural effusion. Respir Med 2015, 109(9):1188-1192.

28. Bielsa S, Martín-Juan J, Porcel JM, Rodríguez-Panadero F: Diagnostic and prognostic implications of pleural adhesions in malignant effusions. J Thorac Oncol 2008, 3(11):1251-1256.

29. Jin F, Wang H, Li Q, Li S, Lai G, Huang J, Huang Y, Jiang T, Bai C, Li S et al: Expert consensus for diagnosis and treatment using medical thoracoscopy in China. J Thorac Dis 2020, 12(5):1799-1810.

30. Wan Y-Y, Zhai C-C, Lin X-S, Yao Z-H, Liu Q-H, Zhu L, Li D-Z, Li X-L, Wang N, Lin D-J: Safety and complications of medical thoracoscopy in the management of pleural diseases. BMC Pulm Med 2019, 19(1):125. 